\title{
A cautionary note on elasticity analyses in ternary plot using randomly generated population matrices.
}

Takenori Takada ${ }^{1, *}$, Yuka Kawai $^{1} \&$ Roberto Salguero-Gómez ${ }^{2,3,5}$

1: Graduate School of Environmental Science, Hokkaido University, Kita-ku, Sapporo 060-0810, Japan.

2: Department of Zoology, Oxford University, New Radcliffe House, Radcliffe Observatory Quarter, 6GG, Woodstock

Rd, Oxford OX2, UK.

3: Centre for Biodiversity and Conservation Science, University of Queensland, St Lucia 4071 QLD, Australia.

4: Evolutionary Demography laboratory, Max Plank Institute for Demographic Research, Rostock 18057, Germany.

5: Department of Animal \& Plant Sciences, University of Sheffield, Alfred Denny Building, Western Bank, Sheffield,

S10 2TN, UK.

${ }^{*}$ Corresponding author: Takenori Takada, E-mail address: takada@ees.hokudai.ac.jp

There are 19 text pages with 4376 words in total, 3 figures, 2 tables, and 1 ESM. 


\section{Abstract}

Matrix population models are one of the most common mathematical models in ecology, which describe the dynamics of stage-structured populations and provide us many population statistics. One of the statistics, elasticity onto population growth rate, is frequently used and represents the degree of the relative impact of life history parameters to the population growth rate. Due to the utility of elasticities for cross-taxonomic comparisons, Silvertown and his coauthors have published multiple papers and reported relationship between elasticities and life forms (or life history) in multiple plant species, using a triangle map (called "ternary plot"). To understand why their elasticities are located in specific regions of the ternary plot, we constructed four archetypes of population matricies, from which we simulated 24,000 randomly-generated population matrices and obtained the consequent elasticities. We found a large discrepancy comparing our results to those in Silvertown's study: for our simulated matrices where rapid transitions were not allowed (e.g. trees), the elasticity distribution resulted in a line across the ternary plot. We provided the mathematical proof for this result, and found that its slope depends on matrix dimension. We also used 1,230 matrices from the COMPADRE Plant Matrix Database and calculated the elasticities. Our simulated results were validated with field data from COMPADRE: two straight lines appeared in the ternary plot. Furthermore, we answered several addressed questions, such as "Is there any special elasticity distribution in matrices with high population growth rates?" and "Why are the elasticities of natural populations concentrated in the upper half of the ternary plot?”. 
Keywords: COMPADRE Plant Matrix Database, comparative biology, population matrix models, demographic simulation, elasticity vector distribution. 


\section{Introduction}

Matrix population models (MPMs hereafter) are one of the most common mathematical models in ecology, which describe the dynamics of stage-structured populations (Caswell 2001; Salguero-Gómez \& de Kroon 2010) as:

$$
\mathbf{x}_{t+1}=\mathbf{A} \mathbf{x}_{t}
$$

where $\mathbf{x}_{t}$ denotes population vector at time $t$ whose element represents the number of individuals at each stage and $\mathbf{A}$ represents population projection matrix. The matrix $\mathbf{A}$ includes important life history parameters in their elements, such as survival probability and fecundity, and provides us many population statistics via calculation of linear-algebraic quantities of $\mathbf{A}$, i.e. population growth rate, stable stage distribution, sensitivity, elasticity or life expectancy (Caswell 2001). Among these, elasticity is a frequently used statistic and given by the relative change in a statistic of the population (e.g. the population growth rate $\lambda$ ) per relative change in matrix elements (Caswell et al. 1984; de Kroon et al. 1986; van Groenendael et al. 1994; Pfister 1998; Kaneko et al. 1999; de Kroon et al. 2000; Cruz-Rodriguez et al. 2009; Kaneko \& Takada 2014). Because it is calculated based on relative changes, the elasticity of population growth rate to matrix elements is formulated as the limit of the ratio of proportional changes in population growth rate $(\lambda)$ to that in matrix elements $\left(a_{i j}\right):$ :

$$
e_{i j}=\lim _{\Delta a_{i j} \rightarrow 0} \frac{\Delta \lambda}{\lambda} / \frac{a_{i j}}{a_{i j}} \frac{\partial \lambda}{\partial a_{i j}}
$$

where $\Delta \lambda$ and $\Delta a_{i j}$ denote the changes in $\lambda$ and $a_{i j}$, respectively. Therefore, $\Delta \lambda / \lambda$ and $\Delta a_{i j} / a_{i j}$ 
means the proportional changes in population growth rate and matrix element. The $e_{i j \mathrm{~s}}$ are normally presented in matrix form and biologically interpreted as the degree of the relative impact of a demographic process expressed by $a_{i j}$ onto the population growth rate. The elements of any elasticity matrix are proven mathematically to satisfy a conservation law whereby the sum of the elasticities over all the elements is equal to unity: $\sum_{i} \sum_{j} e_{i j}=1$ (de Kroon et al. 1986). This property of the elasticity matrix has been used in multiple inter- and intra-specific demographic analyses in plants (Silvertown et al. 1996).

Silvertown and collaborators published a series of papers applying elasticity analysis to plants to classify their life forms. In them, they proposed a scheme of division of the matrix elements into three categories, i.e., fecundity, growth, and stasis (in their case defined as no-change in stage (true stasis) as well as retrogression), and defined three types of elasticities of $\lambda$ : $E_{\text {Fecundity, }} E_{\text {Growth, }}$ and $E_{\text {Stasis }}$ (Silvertown \& Franco 1993; Silvertown et al. 1993; Franco \& Silvertown, 1996; Silvertown et al. 1996; Franco \& Silvertown, 2004). $E_{\text {Fecundity }}$ is the sum of the elasticities of $\lambda$ to all fecundity elements in the MPM, and the others are defined similarly. Under these definitions, the sum of the above three elasticities is equal to 1 because of the aforementioned conservation law. Therefore, the elasticity vector $\boldsymbol{E}=\left(E_{\mathrm{Fecundity}}, E_{\mathrm{Growth}}, E_{\mathrm{Stasis}}\right)$ of any MPM can be plotted on a ternary plot (i.e. two-dimensional simplex in mathematics, Fig.1a) because the lengths of $E_{\text {Fecundity }}, E_{\text {Growth, }}$ and $E_{\text {Stasis }}$ in Fig. 1a sum up to 1 for any point in the right triangle with side 1 long. Silvertown et al. (1996) obtained the elasticity vectors for 84 plant 
species, including trees and herbs, and mapped them on a ternary plot. They showed that the location of a species in the ternary plot, as given by its elasticity vector, depended on its life form (or life history). The authors discussed the relationship between the distribution of the species' vectors and the characteristics of their life form (or life history) (Fig. 1b-1d). Semelparous species were distributed on the upper-left region of the triangle, where growth and fecundity are more important to $\lambda$ than stasis (Fig. 1b), whereas herbs occupied a region from the center toward the $E_{\text {Stasis }}=1$ vertex (Fig. 1c). Importantly woody species, characterized by slow growth, were found within a relatively narrow strip from the vertex $E_{\text {Stasis }}=1$ (Fig. 1d).

Two striking patterns were found using the ternary plot and elasticity vector approach. First, few elasticity vectors were found in the lower half of the triangle, corresponding to species whose $\lambda$ values would depend strongly on stasis and intermediately to fecundity (Fig. 1). Second, most of the regions spread from the lower right vertex, where the population dynamics are fully dominated by stasis. To understand and clarify the reason of the above two patterns, we constructed four archetypes of population matrices in which the elements were randomly generated from two probability distributions ("randomly-generated population matrix (RPM)" hereafter). We examined the distribution of the elasticity vectors of RPMs and answered the following addressed questions: (i) what is the potential range of space occupied by RPMs in the triangle simplex? (ii) Is there any special elasticity distribution in matrices with high population growth rates? (iii) Where are the elasticities located when we assume a different archetype for the RPMs?, and (iv) Why are the elasticity vectors of natural populations distributed in the upper half 
of the triangle?

A comparison of the distributions derived from four archetypes reveals the reason behind the characteristic patterns reported by Silvertown et al. (1996). Furthermore, we obtain the elasticity vectors of data from 1,230 MPMs (166 plant species) in the COMPADRE database (Salguero-Gómez et al. 2015) and show that there are two straight lines in the distribution of elasticity vectors from natural populations. Finally, we answer four addressed questions and discuss the applicability of RPMs in evolutionary ecology and perspectives related to this approach.

\section{Methods}

Elasticity analysis of life cycle archetypes

We constructed multiple $4 \times 4$ randomly-generated population matrices composed of two key demographic processes: fecundity $(f)$ and transition probabilities from a particular stage to another $\left(t_{i j}\right)$. The fecundity part was located on only one matrix element, element $(1,4)$ of the matrix, corresponding to the recruitment into the smallest/least developed/youngest class by reproductive individuals from the largest/most developed/oldest class, as

$$
\mathbf{A}=\left(\begin{array}{cccc}
t_{11} & t_{12} & t_{13} & f \\
t_{21} & t_{22} & t_{23} & t_{24} \\
t_{31} & t_{32} & t_{33} & t_{34} \\
t_{41} & t_{42} & t_{43} & t_{44}
\end{array}\right)
$$

The fecundity $(f)$ was randomly generated using a Poisson distribution with average $p$ : 
$\operatorname{Pr}(f)=\frac{p^{f} \exp (-p)}{f !}$,

where $\operatorname{Pr}(f)$ represents the probability that the fecundity is equal to $f$ and $f$ is a non-negative integer.

"RandomVariate" command in Mathematica 10 (Wolfram Research, Inc.) was used in generating random samples of fecundity and the generated sequence was, e.g., $\{4,2,4,8,8,7,9,6,3,5,7,7$, $2,5,3,7,6,4,6,7, \ldots \ldots\}$ when $p=5$. We assigned the $i$-th element in the sequence to fecundity in $i$-th constructed matrix. The Poisson distributions with $p=5$ and 20 were used in the present paper.

Transition probabilities $\left(t_{i j}\right)$ ranged from $0-1$ and the column sums were constrained to $\leq 1$, reflecting the biological requirement that individuals at a given stage cannot survive over $100 \%$ between time steps. The probabilities at the $j$-th stage $\left(t_{i j}\right)$ were randomly generated using "RandomReal" command in Mathematica 10, e.g. $(0.4459,0.4955,0.1836,0.5721,0.7747)$, and the numbers were normalized dividing them by their sum so that the sum was equal to $1:(0.1804$, $0.2005,0.0743,0.2314,0.3134)$. Then, we assumed the 5-th normalized number $(0.3134)$ was the death probability at the stage and assigned the first four (three when $j=4$ ) numbers to $t_{1 j}, t_{2 j}, t_{3 j}$ and $t_{4 j}\left(t_{2 j}, t_{3 j}\right.$ and $t_{4 j}$ when $\left.j=4\right)$. All the transition probabilities $(j=1$ to 4$)$ were obtained by four-times iteration of the above procedure. An example of randomly generated population matrix (RPM) is as follows:

$$
\mathbf{A}=\left(\begin{array}{cccc}
0.1804 & 0.0150 & 0.2004 & 4 \\
0.2005 & 0.1944 & 0.3353 & 0.2968 \\
0.0743 & 0.3687 & 0.2868 & 0.5106 \\
0.2314 & 0.1057 & 0.1261 & 0.0330
\end{array}\right)
$$


In order to explore the elasticity vector distribution in ternary space that can be generated by this type of RPM, we constructed the following four archetypes:

Archetype I: The matrix of this type $\mathbf{A}_{1}$ has the same form as Eq. (3), where $f>0,0<t_{i j}<1, \sum_{i} t_{i j}<1$. All elements are positive, although they may be very small. This RPM approximates a life history where individuals can progress and retrogress rapidly.

Archetype II: $\mathbf{A}_{2}$ has the same form as archetype I, i.e. Eq. (3), where $f>0,0<t_{i j}<1, \sum_{i} t_{i j}<1, \sum_{i} t_{i j}<\sum_{i} t_{i, j+1}$. Since $\sum_{i} t_{i j}$ represents the survival probability at $j$-th stage, the fourth inequality biologically means that the survival probability increases monotonously as the individuals advance to later stages. This RPM, as the one in the first archetype, also allows for rapid progression and retrogression, and is more realistic in that it scales up survival with size/age/stage (e.g. Harper 1977).

Archetype III: $\mathbf{A}_{3}=\left(\begin{array}{cccc}t_{11} & 0 & 0 & f \\ t_{21} & t_{22} & 0 & 0 \\ 0 & t_{32} & t_{33} & 0 \\ 0 & 0 & t_{43} & t_{44}\end{array}\right)$,

where $f>0,0 \leq t_{i j}<1, \sum_{i} t_{i j}<1$. We assumed in matrix $\mathbf{A}_{3}$ that the positive elements are assigned only on element $(1,4)$ and the diagonal and lower sub-diagonal elements. This RPM represents the life cycle of a species where retrogression is not allowed, and progression can only happen to the immediately larger/more developed stage (slow progression, e.g. trees).

Archetype IV: $\mathbf{A}_{4}$ has the same general form as archetype III and Eq. (3), where 
$f>0,0<t_{i j}<1, \sum_{i} t_{i j}<1$. We made the further assumption that $\sum_{i} t_{i j}<\sum_{i} t_{i, j+1}$, as in archetype II. Therefore, the two assumptions of archetypes II and III are combined in $\mathbf{A}_{4}$. This RPM represents a more realistic case than the previous one in that survival increases with size/developmental stage.

We generated 3,000 RPMs for each of the four archetypes and $p=5,20$ (24,000 matrices), and calculated the elasticities,

$$
\left(\begin{array}{llll}
e_{11} & e_{12} & e_{13} & e_{14} \\
e_{21} & e_{22} & e_{23} & e_{24} \\
e_{31} & e_{32} & e_{33} & e_{34} \\
e_{41} & e_{42} & e_{43} & e_{44}
\end{array}\right)
$$

using the formula of elasticity (Caswell 2001):

$$
e_{i j}=\frac{v_{i} u_{j}}{\sum_{k} v_{k} u_{k}},
$$

where $v_{i}$ (or $u_{j}$ ) represents the $i$-th (or the $j$-th) element of left (or right) eigenvector of each RPM. These 16 elasticities were summarized to a vector $\boldsymbol{E}=\left(E_{\text {Fecundity }}, E_{\text {Growth }}, E_{\text {Stasis }}\right)$ following a way as Silvertown et al. (1996) assumed: assigning element $(1,4)$ to the fecundity category $(F)$, the diagonal elements and retrogression elements to the stasis category $(S)$, and the lower triangle elements to the growth category $(G)$, i.e.,

$$
\left(\begin{array}{llll}
S & S & S & F \\
G & S & S & S \\
G & G & S & S \\
G & G & G & S
\end{array}\right) .
$$


Therefore,

$$
\begin{array}{ccc}
E_{\text {Fecundity }} & = & e_{14} \\
E_{\text {Growth }} & = & e_{21}+e_{31}+e_{32}+e_{41}+e_{42}+e_{43} \\
E_{\text {Stasis }} & = & e_{11}+e_{12}+e_{13}+e_{22}+e_{23}+e_{24}+e_{33}+e_{34}+e_{44}
\end{array} .
$$

The 3000 points of elasticity vectors $\boldsymbol{E}$ of the RPMs were plotted and distributed in a ternary plot (two-dimensional plane), as described in Fig. 1. Eight distributions of the vectors were obtained in all: $p=5$ and 20 in archetype $\mathbf{A}_{1} ; p=5$ and 20 in archetype $\mathbf{A}_{2} ; p=5$ and 20 in archetype $\mathbf{A}_{3} ; p$ $=5$ and 20 in archetype $\left.\mathbf{A}_{4}\right)$.

We obtained several statistics of elasticity vector distribution to estimate the effect of population growth rate $(\lambda ;$ Table 1a), levels of reproduction ( $p$; Table 1b) and archetype (Table 1c) on the distributions. The statistics are the mean and the standard deviation of each distribution and Sørenson distance index between two distributions, which were used to compare two distributions. The mean were used to estimate how much the center of the distribution shifts between the two distributions. Sørenson distance index $\left(\mathrm{S}_{\text {index }}\right)$ is a measure used to estimate the degree of overlap of two distributions. To calculate $\mathrm{S}_{\text {index }} \mathrm{S}$, we made a polygon for each elasticity vector distribution, which is a region including all the distributed points inside the closed curve, as shown in Fig.2a. Then, two polygons were obtained from two elasticity vector distributions to be compared (Fig.2b) and $\mathrm{S}_{\text {index }}$ of the two polygons was calculated using the definition of $2 x /(2 x+y+z)$, where $x$, $y$ and $z$ represent the overlap area of the first and the second polygons, the area unique to the first polygon and the area unique to the second polygon, respectively (Fig. 2b). It is equal to 1 when two areas are the same $(y=z=0)$ and equal to zero when they are not overlapped. $\mathrm{S}_{\text {index }}$ can be 
used to estimate how much the whole distributions differ. The comparison was made in three ways as follows: between the distributions for $\lambda>1$ vs. $\lambda<1$, between the distributions for $p$ $=5$ vs. 20 in each archetype, and between the distributions of archetypes I vs. II.

Furthermore, we obtained a mathematical formula on the elasticity vectors analytically in archetypes III and IV and proved mathematically that the distribution should lies on a line. Furthermore, we obtained a formula to determine the angle $(\theta)$ of the straight line (see ESM S1).

\section{Elasticity analysis of natural populations}

We sampled MPMs from the COMPADRE Plant Matrix Database (Salguero-Gómez et al. 2015) to obtain the elasticity vectors. COMPADRE (ver. 4.0.1) contains 4,246 “individual” MPM from 695 plant species. Each individual MPM describes the dynamics of a given population at single site and study time-step for a given treatment. To retain MPMs of the highest quality, we applied a series of selection criteria: (a) only MPMs where the stage-specific survival rates are $\leq 1$.; (b) populations where fecundity is explicitly measured; (c) populations parameterized as "unmanipulated" treatment conditions (i.e., controls), (d) MPMs with a sampling periodicity = 1, which means that the time step in calculating the population dynamics is one year to allow for cross-taxonomic comparisons of perturbation of $\lambda$ on the same basis; and (e) MPMs that are irreducible and primitive to be able to calculate elasticities. In biological terms, a reducible matrix means that the life cycle contains at least one stage that cannot contribute, by any developmental path, to some other stage(s). Otherwise, the matrix is irreducible (Caswell 2001). In primitive 
matrices, the stable stage distribution is realized in the long-term dynamics and periodical behavior of the dynamics does not exist (Caswell 2001). This criterion ensures that the elements of the sensitivity matrix are all positive; (f) MPMs with seed stages were excluded because of the considerable uncertainty in their parameter estimation in field conditions, as the inclusion of a seed stage can result in an artifactual year gap (Caswell 2001; Salguero-Gómez et al. 2015). For criterion (d), the irreducibility of each MPM was checked by examining whether $(\mathbf{I}+\mathbf{A})^{n-1}$ results in a positive matrix using a theorem proposed by Horn \& Johnson (1985), where $n$ and I represent the matrix dimension of $\mathbf{A}$ and the identity matrix, respectively. The primitivity of a given MPM was checked by examining whether $\mathbf{A}^{n^{2}-2 n+2}$ is a positive matrix.

Our set of selection criteria applied to COMPADRE resulted in 1,230 MPMs from 166

plant species. The elasticity vectors of these MPMs were plotted in the triangle simplex, as were those generated from the RPMs, which then allowed us to examine the full space of elasticity vectors that can be explored by a RPM versus those found in nature.

\section{Results}

Elasticity analysis of life cycle archetypes

In archetype I, representing a life-cycle where only $4^{\text {th }}$ stage individuals can reproduce and where individuals can progress and retrogress rapidly but without a relationship between survival 
and stage number, the distribution of elasticity vectors was concentrated in the upper-center region of the ternary plot (Fig. 3a \& 3b), differing from the overall result reported by Silvertown et al. (1996; Fig. 1). Specifically, our distribution shifted to the upper-left region (i.e. greater importance for fecundity and growth to $\lambda$, lower for stasis) when we increased the average fecundity ( $p=20$; Fig. $3 \mathrm{~b}$ and Table 1b). The overlapping index by Sørenson of the distributions in Fig. $3 \mathrm{a}$ and $3 \mathrm{~b}$ is $62.0 \%$. The average fecundity affected the number of points resulting in values of $\lambda$ above or below. This is due to the fact that increasing fecundity leads to more recruits in the population. Additionally, the classification of RPMs into two groups, depending on whether the population growth rate $\lambda>$ or $<1$, provides another useful insight: the distributions of archetypes I moved towards the lower-right region when $\lambda<1$ and the overlap between the $\lambda>1$ distribution and the $\lambda$ $<1$ distribution is $46.3 \%$ (Fig. 3a, Table 1a).

Archetype II occupied a similar area to archetype I. In archetype II, simulated RPMs followed the same assumptions as in archetype I, but in them survival probabilities increased with stage development. The resulting distributions are shown in Fig. $3 \mathrm{c}$ and $3 \mathrm{~d}$ and the distribution shifted to the upper-left region when we increased the average fecundity ( $p=20$; Fig. 3d). The overlap of the distributions in Fig. $3 \mathrm{c}$ and $3 \mathrm{~d}$ is $55.3 \%$ as shown in Table $1 \mathrm{~b}$. Additionally, the classification of RPMs into $\lambda>1$ and $\lambda<1$ groups affected the distributions of archetypes II, shifting towards the lower-right region when $\lambda<1$. The overlap between the $\lambda>1$ distribution and the $\lambda<1$ distribution is $46.2 \%$ (Fig. 3c, Table 1a).

From the comparison between archetypes I and II, the mean of the distribution tended 
slightly (not large) towards an area of relative greater impact by stasis (bottom-right corner) compared to the area occupied by archetype I (Table 1c). The overlap area in archetypes I and II is high, as depicted by a Sørenson index of $91.3 \%(p=5)$ and $93.9 \%(p=20)$ between both distributions.

The distributions of the elasticity vectors of archetype III and IV are strikingly different from those by archetypes I and II. The main difference of the assumption between both sets of archetypes is that in III and IV, individuals were not allowed to retrogress, nor to progress further than to its immediately superior stage (e.g. rapid progression was not possible; Eq. 4). The elasticity vectors in archetypes III and IV produced a straight line departing from the lower-right corner, where the relative impact of stasis on population growth rate $\lambda$ is the highest possible $\left(E_{\mathrm{S}}=\right.$ 1), and at an angle $\theta=46^{\circ}$ (Fig. 3e to $3 \mathrm{~h}$ ). Comparing the distributions in archetype III depending on whether the population growth rate $\lambda>$ or $<1$, the two distributions were found to be similar (Fig. 3e and 3f). The line moved slightly to lower-right corner when $\lambda$ is less than 1 . The overlap as per Sørenson index was high: $93.5 \%(p=5)$ and $90.8 \%(p=20)$ (Table 1a). It should be noted that we calculated the overlapping length of two straight lines as one-dimensional Sørenson distance index. The distribution in archetype IV had a similar tendency to in archetype III (Fig. $3 \mathrm{~g}$ and 3h, Table 1a and b). Moreover, the spread of the line was greater for archetype III than for archetypes IV; the length of the respective distributions was 0.813 and 0.672 (Fig. 3e and 3g), and 0.845 and 0.726 (Fig. 3f and 3h).

Given the striking linear pattern of archetypes III and IV, we obtained a formula on the 
elasticity vectors analytically:

$$
E_{\text {Fecundity }}=\frac{1}{D \lambda}, E_{\text {Growth }}=\frac{(n-1)}{D \lambda} \text { and } E_{\text {Stasis }}=1-\frac{n}{D \lambda}
$$

where $n$ is the matrix dimension and $D=\sum_{k} \frac{1}{\lambda-t_{k k}}$. Note that the elasticity of growth element $(i+1, i)$ is $e_{i+1, i}=\frac{1}{D \lambda}$ and independent of $i$, which means all the values are the same and are the same as $E_{\text {fecundity }}($ see ESM M1). Since the elements of elasticity vector depend on only one term, $\mathrm{D} \lambda$, the degree of freedom of elasticity vectors is 1 . Therefore, the elasticity vector distribution lies on a straight line, varying the value of $\mathrm{D} \lambda$. Eliminating $\mathrm{D} \lambda$ from Eq. (9), a formula to determine the angle $(\theta)$ of the straight line can be obtained as

$$
\tan \theta=\frac{(n-1) \sqrt{3}}{(n+1)}
$$

We note that in all four archetypes here examined, the number of stages in the life cycles were 4 , thus $n=4$, and $\tan 46^{\circ}=3 \sqrt{3} / 5$.

\section{Elasticity analysis of natural populations}

The elasticity vectors of the 1,230 MPMs selected from the COMPADRE database represent the relative importance of the demographic processes for each species under natural conditions, and the covariance of vital rates that incorporates biological trade-offs among those functions. Keeping these natural restrictions in mind, one can observe that the elasticity vectors of these natural populations lay mostly in the upper half of the triangle simplex (Fig. 4a). This means 
that most of the examined populations are relatively dominated by growth and stasis (with retrogression, see Eq. (7)), but not as much by fecundity. We also found two straight lines departing from the lower-right region running towards the center of the ternary space, with slopes of $30^{\circ}$ and $41^{\circ}$, respectively (Fig. 4b). From Eq. (10), these angles are given by matrices with dimensions of 2 and 3 , respectively:

$$
\tan 30^{\circ}=\frac{(2-1) \sqrt{3}}{(2+1)}=\frac{\sqrt{3}}{3} \text { and } \tan 41^{\circ}=\frac{(3-1) \sqrt{3}}{(3+1)}=\frac{\sqrt{3}}{2} \text {. }
$$

We examined how many MPMs from the selected 1,230 matrices had the same form as in Eq. (4), i.e., a single fecundity element and diagonal and lower sub-diagonal elements are not equal to zero. There were 412 matrices, including 332 - 2 x 2 MPMs and 33-3 x 3 MPMs (Table 2). The elasticity vector of $33.5 \%$ of 1230 MPMs was fallen on those straight lines. Note that all 2 by 2 matrices have the same form as Eq. (3).

\section{Discussion}

We obtained the elasticity vectors ( $\left.E_{\mathrm{Fecundity}}, E_{\text {Growth, }}, E_{\text {Stasis }}\right)$ from four archetypes of RPMs to compare them to those obtained and represented in a ternary plot from the MPMs of 84 plant species in Silvertown et al. (1996), where they made an effort to understand the main drivers behind the location of species on the ternary plot. We next validated our results with an unprecedented number of MPMs from natural populations worldwide to answer questions (i)-(iv) in the Introduction, also detailed below: 
(i) What is the potential range of space occupied by RPMs in the triangle simplex?

The distribution of the elasticity vectors in RPMs of $\mathbf{A}_{1}$ (archetype I) was plotted in the upper-center region and had a large discrepancy from that of natural populations in Silvertown's study (1996). In Fig. 3a and 3b, our distribution was mostly concentrated in a sparse region of the distribution found by Silverton's and his collaborators and dominated by growth (Fig. 1). This discrepancy is expected because RPMs of archetype I were constructed only considering minimum biological assumptions and/or requirements, such as non-negative matrix elements and the column sums being less than 1. To understand the reason for the discrepancy, one of the plausible biological requirements could be that more developed individuals do tend to survive better because they may be more tolerant to environmental stresses and/or may be better competitors (Harper 1977). However, when we explicitly considered this biological requirement, as we did in archetype II, we still found a similar result to that of archetype I (Fig. 3c and 3d). Therefore, we conclude that the difference in assumptions between archetypes I and II is not the main reason for the difference between the distributions reported here and those in Silvertown et al. (1996; Fig. 1).

(ii) Is there any special elasticity distribution in matrices with high population growth rates?

Our approach, producing RPMs constrained to a series of assumptions, can be thought of a simulation exercise where mutations enter a specific population. Most of the mutations would be driven out in the process of natural selection because the fitness is less than 1 . Successful 
mutations alter the fitness of the individuals therein, and consequently the overall population growth rate. The mutations would then determine where species are located on the ternary plot, and what optimal relationships of investments on fecundity, growth and stasis are optimal for natural selection. Ultimately, the essential cause of being favored by natural selection is that the population-average fitness (i.e., the population growth rate) is more than 1 (Takada 1995; Chapter 11 in Caswell 2001; Geyer et al. 2007). Where are the elasticity vectors that correspond to RPMs with $\lambda>1$ located in the ternary plot? We found some general support to a unique answer to this question. The elasticity vector distribution of RPMs moved to the upper-left region of the ternary plot when the population growth rate was greater than 1 in both Fig. 3a and 3c. Furthermore, the distribution of elasticity vectors migrated to the upper-left region of the ternary plot, with an increase impact of fecundity on the population growth rate, when comparing Fig. 3a and $3 \mathrm{~b}$ (3c and 3d). This means that both the relative impacts of fecundity and growth to the overall population dynamics increase with an increase in fecundity. The same tendency was evidenced by our results in Fig. 3e-3h. However, the distribution of elasticity vectors of plants from natural settings in COMPADRE rarely occupies the upper-left region (Figs. 1 and 4a). Instead, these are located in the lower-right region. Before we conducted simulated calculation on RPMs, we expected that there must be some evolutionary force for the bias in elasticity distribution of natural plant populations. The first candidate of the evolutionary force we considered was population viability; whether the population growth rate is greater than 1 or not. However, the viability shifted the distribution toward upper-left region, which is the opposite side of the distribution in natural 
populations. Therefore, the viability in plant populations is not the reason for interpreting the elasticity distribution of real plants.

(iii) Where are the elasticities located when we assume different archetypes of RPMs?

Under the assumption that most plants in nature do not grow fast, as it happens in trees (Hartshorn 1975; Platt et al. 1988; van Mantgem \& Stephenson 2005; Lin \&Augspurger 2008; Münzbergová et al. 2013; Salguero-Gómez et al. 2016), i.e., the RPMs given by Eq. (4), the elasticity distribution lay on a line with a slope of $46^{\circ}$ (Fig. 3e and 3f). The region of elasticity vectors had a similar distribution to that of woodland herbs, shrubs, and trees (Fig. 1c and 1d). We have proven mathematically here that matrices with the special structure of Eq. (4) result in a linear distribution of elasticity vectors (see ESM S1). Not coincidentally, two straight lines were found in the triangle map obtained from the population matrices of COMPADRE (Fig. 4), which explained $35.1 \%$ of the 1230 plant species (432 species). If the assumption that most plants do not grow quickly is "approximately" correct in the remaining $64.9 \%$ of plants, the distribution of elasticity vectors would be scattered around a straight line depending on the matrix dimension $n$ (see Eq. (10)). Therefore, the distributions in Figs. 1 and 4a could be the assemblage of several distributions around several straight lines (Fig. 4c). We speculate that the special structure of projection matrices, i.e., the assumption of not growing quickly, is one of the important reasons for the observed elasticity distribution. It will be an interesting subject to explore whether the assumption in archetypes III and IV holds "approximately" in the remaining $64.9 \%$ of plants. 
(iv) Why are the elasticity vectors of natural populations distributed in the upper half of the

triangle?

The slope of the linear distribution of elasticity vectors depends on the dimension of the projection matrix, as in Eq. (10). When the dimension is 2, the slope is $30^{\circ}$ (Fig. 4b). As $n$ approaches $\infty$,

$$
\tan \theta=\lim _{n \rightarrow \infty} \frac{(n-1) \sqrt{3}}{(n+1)}=\sqrt{3}
$$

and the angle tends to $60^{\circ}$. Therefore, the elasticity distribution obtained from projection matrices with a variety of matrix dimensions would be distributed between lines with slopes of $30^{\circ}$ and $60^{\circ}$ (Fig. 4c). Therefore, most of the elasticity vectors of natural populations would be distributed in the upper half of the triangle.

When validating our simulations with MPMs from COMPADRE, which were carefully chosen to represent natural settings under control conditions, we found that there were two straight lines in ternary plot. The result clarified that the distribution of elasticity vectors strongly depends on matrix dimension and structure (e.g. how many and where are zero elements positioned in the MPM in question and into which stages newly-born offspring are recruited). Therefore, we call for a more cautious interpretation of ternary plots of elasticity, specifically in regards to slow-living creatures such as trees, or to age-based MPMs. It is mathematically proven that the elasticity 
distribution in age-based MPMs lies on a straight line in ternary plot (see ESM1 (iii)). Furthermore, in comparative study using the matrix database, we should be more careful in determining selection criteria for appropriate matrices although the selection criteria depend on the purpose of each study. Specifically, species with clonal reproduction occasionally show a diverse matrix structure, i.e. the newly born individuals through clonal reproduction are recruited into a variety of stages.

Additionally, the variation in matrix dimensions of MPMs should be paid attention to when we use the ternary plot in comparing multiple species.

Several authors have pointed out that the matrix dimension affects the results of demographic statistics such as population growth rates and elasticities (Silvertown et al. 1993; Enright et al. 1995; Lamar \& McGraw 2005; Ramula \& Lehtila 2005; Salguero-G6mez \& Casper 2010; Salguero-Gómez \& Plotkin 2010). If the structure had the form in Eq. (4), the variation in elasticity vectors would occur along a line from the lower-right corner to the edge of the $E_{\text {growth }}$ axis in the upper half of the triangle simplex. Where along this line is the elasticity vector located for a specific demography? This question concerns the very evolution of a plant's life history, and remains unsolved theoretically. We expect this would be an interesting subject to be solved in future. 


\section{Acknowledgments}

We sincerely thank Dr. Motohide Seki and Yuya Tachiki for their valuable

suggestions. We thank the Max Planck Institute for Demographic Research for the development and curation of the COMPADRE Plant Matrix Database. TT was supported in part by JSPS KAKENHI (Grant Numbers JP26291087 and JP15H04418). RSG was supported by the Australian Research Council (DE140100505) and the UK NERC (R/142195-11-1) 


\section{References}

2 Caswell H, Naiman R, Morin R. (1984) Evaluating the consequences of reproduction in

3 complex salmonid life cycles. Aquaculture 43:123-143

4 Caswell H. (2001) Matrix population models. John Wiley \& Sons, Ltd. DOI 10.1002/9781118445112.stat07481

6 Cruz-Rodriguez JA, Lopez-Mata L, Varverde T (2009) A comparison of traditional elasticity and variance-standardized perturbation analyses: a case study with the tropical tree species Manilkara zapota (Sapotaceae). J Trop Ecol 25: 135-146

9 de Kroon H, Plaisier A, van Groenendael J, Caswell H. (1986). Elasticity: the relative 10 contribution of demographic parameters to population growth rate. Ecology, 67, $1427-1431$.

12 de Kroon H, van Groenendael J, Ehrlén J. (2000) Elasticities: a review of methods and model 13 limitations. Ecology 81: 607-618.

14 Enright NJ, Franco M, Silvertown J. (1995) Comparing plant life histories using elasticity 15 analysis: the importance of life span and the number of life cycle stages. Oecologia 104:79-84.

17 Franco M, Silvertown J. (1996) Life history variation in plants: an exploration of the fastslow continuum hypothesis. Philos Trans R Soc Lond B, 351:1341-1348.

19 Franco M, Silvertown J. (2004). A comparative demography of plants based upon elasticities of vital rates. Ecology, 85: 531-538. 
21 Geyer, CJ, Wagenius, S, Shaw, RG. (2007). Aster models for life history analysis. Biometrika, 94: 415-426.

23 Harper, JL. (1977) Population biology of plants. Academic Press, New York.

24 Hartshorn GS (1975) A matrix model of tree population dynamics. In: Golley FB, Medina E (eds) Tropical ecological systems. Spring-Verlag, New York, pp. 41-51

Horn R, Johnson C. (1985) Matrix Algebra, Cambridge University, Cambridge, UK.

Kaneko Y, Takada T, Kawano S (1999) Population biology of Aesculus turbinata Blume: a demographic analysis using transition matrices on a natural population along a riparian environmental gradient. Plant Species Biol 14: 47-68

Kaneko, Y, Takada T. (2014). Pair-wise analyses of the effects of demographic processes, vital rates, and life stages on the spatiotemporal variation in the population dynamics of the riparian tree Aesculus turbinata Blume. Pop Ecol, 56: 161-173.

Lamar WR, McGraw JB. (2005) Evaluating the use of remotely sensed data in matrix 212:50-64.

Lin, Y, Augspurger, CK. (2008). Impact of spatial heterogeneity of neighborhoods on

Münzbergová, Z, Hadincová, V, Wild, J, Kindlmannová, J. (2013). Variability in the long-term population dynamics of sugar maple (Acer saccharum). For Ecol Manage, 255: 3589-3596. 

invasion success of Pinus strobus. Plos One, 8, e56953.

42 Pfister CA (1998) Patterns of variance in stage-structured populations: evolutionary predictions and ecological implications. Proc Natl Acad Sci USA, 95: 213-218.

44 Platt, WJ, Evans, GW, Rathbun, S. (1988). The population dynamics of a long-lived conifer (Pinus palustris). Am Nat, 131: 491-525.

Ramula S, Lehtila K. (2005) Matrix dimensionality in demographic analysis of plants: when to use smaller matrices? Oikos 111:563-573.

Salguero-Gómez R, Casper BB. (2010). Keeping shrinkage in the demographic loop. J Ecol 98:312-323.

Salguero-Gómez R, de Kroon H. (2010). Matrix projection models meet variation in the real world. J Ecol, 98: 250-254.

Salguero-Gómez R, Plotkin JB. (2010) Matrix dimensions bias demographic inferences: implications for comparative plant demography. Am Nat, 176: 710-722.

Salguero-Gómez R, Jones OR, Archer CR, Buckley YM, Che-Castaldo J, Caswell H, ... and for plant demography. J Ecol 103: 202-218 DOI 10.1111/1365-2745.12334

Salguero-Gómez, R, Jones, OR, Jongejans, E, Blomberg, SP, Hodgson, DJ, Mbeau-Ache, C, ... Buckley, YM. (2016). Fast-slow continuum and reproductive strategies structure plant life-history variation worldwide. Proc 
61 Silvertown J, Franco M. (1993) Plant demography and habitat: a comparative approach. Plant Species Biol 8: 67-73.

63 Silvertown J, Franco M, Pisanty I, Mendoza A. (1993). Comparative plant 64 demography--relative importance of life-cycle components to the finite rate of increase in woody and herbaceous perennials. J Ecol, 465-476.

66 Silvertown J, Franco M, Menges E. (1996) Interpretation of elasticity matrices as an aid to 67 the management of plant populations for conservation. Conserv Biol 10: 591-597.

68 Takada, T. (1995). Evolution of semelparous and iteroparous perennial plants::

69 Comparison between the density-independent and density-dependent dynamics. J Theor Biol, 173: 51-60. van Groenendael J, de Kroon H, Kalisz S, Tuljapurkar S. (1994) Loop analysis: evaluating life history pathways in population projection matrices. Ecology, 75: 2410-2415. van Mantgem, PJ, Stephenson, NL. (2005). The accuracy of matrix population model projections for coniferous trees in the Sierra Nevada, California. J Ecol 93: 737-747. 


\section{$77 \quad$ Figure legends}

78 Figure 1. Ternary plot (= triangle simplex) of the elasticity vector $(\boldsymbol{E})$ of three main

79 demographic processes: fecundity $(F)$, growth $(G)$ and stasis $(S)$ for 84 wild plant species'

80 populations (redrawn from Fig. 2 in Silvertown et al. 1996). (a) Coordinates of elasticity

81 vector $\boldsymbol{E}=\left(E_{\text {Fecundity }}, E_{\text {Growth }}, E_{\text {Stasis }}\right)$ in ternary plot. $E_{\text {Fecundity }}+E_{\text {Growth }}+E_{\text {Stasis }}=E_{\text {Fecundity }}+$ "o"

$82+$ "||" = 1 because of the right triangle, (b) Area occupied by semelparous species, (c) by

83 herbs in open habitats (hatched) and in forest habitats (white), and (d) by shrubs and trees.

84 Note that the area occupied by herbs in open and close habitats overlaps.

86 Figure 2. Polygon and Sørenson distance index.

87 (a) A polygon can be drawn for an elasticity vector distribution which has 3000 points

88 (small "+" marks). It is a region including all the distributed points inside the closed curve.

89 (b) Two polygons obtained from two elasticity vector distributions are composed of three

90 areas: the overlap area of the first and the second polygons, the area unique to the first

91 polygon and the area unique to the second polygon. Their areas are $x, y$ and $z$, respectively.

92 Sørenson distance index ( $\left.\mathrm{S}_{\text {index }}\right)$ is calculated as $2 x /(2 x+y+z)$.

94 Figure 3. Ternary plot of elasticityvector $(\boldsymbol{E})$ of fecundity $(\mathrm{F})$, growth (G) and stasis (S) for

95 3,000 simulated 4-stage matrix population models for each of the four inspected archetype

96 life cycles: archetype I: rapid progression and retrogression are allowed (See equation 3); 
97 archetype II: same as archetype I, but survival increases with stage number; archetype III:

98 retrogression does not occur, and progression can only happen to the immediately superior

99 class, mimicking the demography of slow-living creatures like trees; archetype IV: same as

100 III, but survival increases with stage number. Blue (red) dots correspond to simulated models

101 where the emerging population growth rate is $\lambda<1(>1)$. The blue (red) dot with solid line

102 represents the mean of elasticity vector for $\lambda<1(>1)$. Ternary plots are presented for two

103 levels of reproduction: $p=5$ (left) and (b) $p=20$ (right). Note that for archetypes III and IV

104 the distribution on the triangle simplex spread along a straight line departing from $E_{\mathrm{S}}=1$ with 105 an angle $\theta=46^{\circ}$.

107 Figure 4. Ternary plot of elasticity vector $(\boldsymbol{E})$ of fecundity (F), growth (G) and stasis (S) for

1081,230 matrix population models (MPMs) from 166 plant species in the COMPADRE 109 database, reporting population dynamics in the wild under unmanipulated conditions. Two

110 straight lines can be observed: one in the lower-right region and another in the center of the

111 ternary plot. The slopes of these lines correspond to matrix dimensions 2 and 3, as per Eq.

112 (10). (a) Original distribution of the examined species from COMPADRE, (b) Two dashed

113 lines with slopes of $30^{\circ}$ (blue) and $41^{\circ}$ (red) are added to (a). (c) Conceptual panel showing

114 five straight lines and scattered distributions around each line for a given matrix dimension $n$,

115 ranging from two to five. 